\title{
Heart Valve Cage Device
}

National Cancer Institute

\section{Source}

National Cancer Institute. Heart Valve Cage Device. NCI Thesaurus. Code C49983.

The part of a mechanical heart valve designed to contain and guide the occluder ball in a cage and ball valve. 